\title{
Correction of Color Cast Images using Histogram Equalization of Local Area
}

\author{
Makoto MISUMI ${ }^{\text {a, }}$, Hideaki ORII a ${ }^{\text {, Tanjina SHARMIN }}{ }^{\text {a }}$, Kenji MISHIMA ${ }^{\text {a }}$ \\ ${ }^{a}$ Faculty of Engineering, Fukuoka University, 8-19-1 Nanakuma, Jonan-ku, Fukuoka 814-0180, JAPAN
}

*Corresponding Author: mmisumi@fukuoka-u.ac.jp

\begin{abstract}
Taking a picture is very popular because of the spread of mobile phones. When the photo was taken in the morning or evening, the star couldn't be visible by the gradation of brightness. In such images, it is difficult to recognize the stars by the image processing because of they have the star in bright area and dark area. In this paper, we propose a method to improve the visibility of the stars using histogram equalization of local area of such images. In the experiment, it was confirmed to improve the visibility by applying the proposed method to the image having the light gradation.
\end{abstract}

Keywords: Color Cast, Histogram Equalization, Local Area.

\section{Introduction}

Taking a picture is very popular because of the spread of device having a digital camera features such as a mobile phone. Recent digital cameras have functions of exposure adjustment and a white balance correction [1-5]. However, exposure adjustment and white balance correction for the sunrise and sunset photos is difficult because of these photos have the brightness of the gradient. Such as sunset and sunrise photograph, an image affected by the particular color tint is called the color cast images. The Fig.1(a) is an image of star with a color cast. The Fig.1(b) is an image that has been color cast correction the entire area of the Fig.1(a). As shown in the image Fig.1(b), the lower portion of the image has become white. Whitish area is similar to the brightness of the background color and stars. When the brightness of the star and the background color is similar it is difficult to find stars by image processing. Whitish area occurs when the color cast correction to the entire image with the brightness

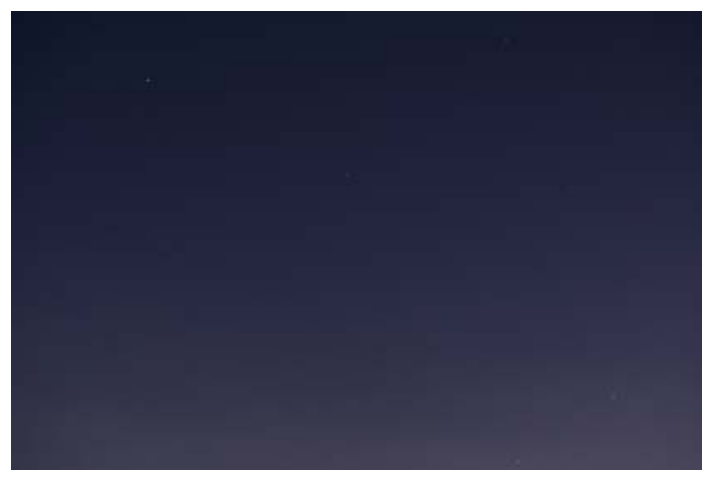

(a) Color cast image

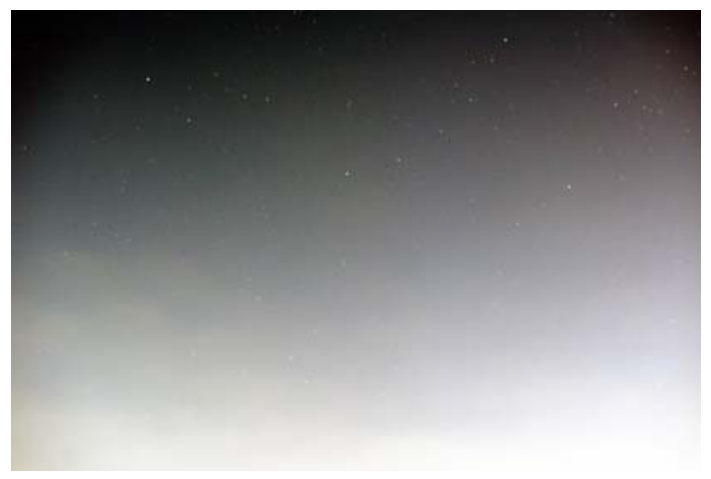

(b) Corrected image (entire area)

Fig. 1. Color cast correction

of the gradient. For the above reasons, when detection of star is objective, the use of correction of color cast image of entire area are not suitable.

In this study, we propose the method to apply the color cast correction for the local area that is similar in brightness value. In the proposed method, the local region is determined by the floodfill algorithm, then color cast of the local area is corrected by the use of histogram equalization. 


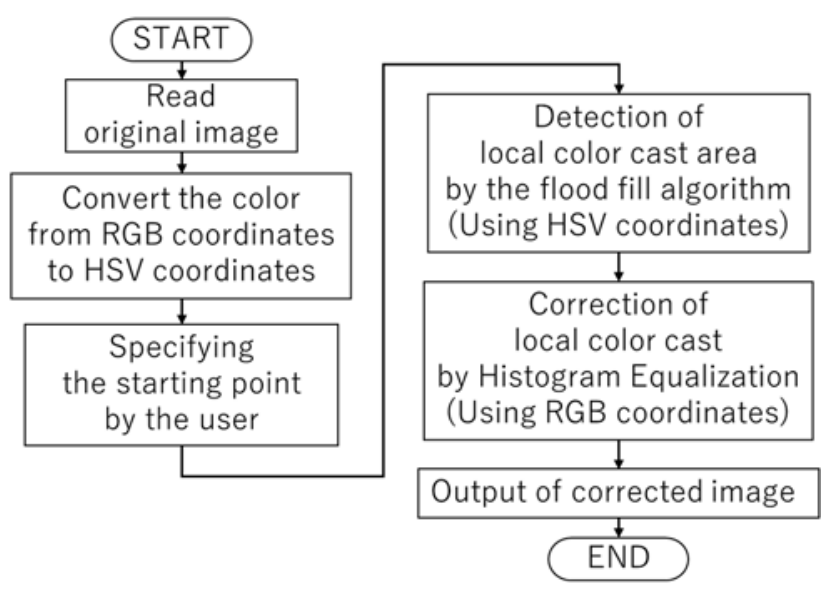

Fig. 2. Flow chart of correction of color cast in local area.

\section{Proposed Method}

A flow chart of correction of color cast in local area for a color photo is shown in Fig.2. We applied histogram equalization for the fog color and the flood fill algorithm in the HSV color space to correction of color cast in local area. In Section 2.1, we describe Histogram Equalization to be used for correction of the fog color. In section 2.2, we describe the conversion of a color space for the detection of the local area.

\subsection{Histogram Equalization}

Histogram equalization [6-10] is a technique for adjusting image intensities to enhance contrast.

$f$ is represented as $m_{r}$ by $m_{c}$ matrix of integer pixel intensity from 0 to $L-1$, and that the specified image. $L$ is, the number of possible intensity values, is 256 in many cases. $p$ shall indicate a normalized histogram of $f$ at the bin for each possible intensity. So $p_{n}$ it will be represented by the following equation.

$$
\begin{aligned}
& p_{n}=\frac{\text { number of pixels with intensity } n}{\text { total number of pixels }} \\
& \qquad(n=0,1, \ldots, L-1)
\end{aligned}
$$

The histogram equalized image $g$ will be defined by following equation, where floor() rounds down to the nearest integer.

$$
g_{i, j}=\operatorname{floor}\left((L-1) \sum_{n=0}^{f_{i, j}} p_{n}\right)
$$

This is equivalent to transforming the pixel intensities, $k$ of $f$ by the function $T(k)$.

$$
T(k)=\operatorname{floor}\left((L-1) \sum_{n=0}^{k} p_{n}\right)
$$

The motivation for this transformation comes from thinking of the intensities of $\mathrm{f}$ and $\mathrm{g}$ as continuous random variables $X, Y$ on $[0, L-1]$ with $\mathrm{Y}$ defined Equation 4 , where $p_{x}$ is the probability density function of $f$.

$$
Y=T(X)=(L-1) \int_{0}^{X} p_{n}(x) d x
$$

$T$ is the cumulative distributive function of $X$ multiplied by $(L-1)$. Assume for simplicity that $T$ is differentiable and invertible. It can then be shown that $Y$ defined by $T(X)$ is uniformly distributed on $[0, L-1]$, namely that $p_{Y}(y)=$ $\frac{1}{L-1}$.

$$
\begin{aligned}
& \begin{aligned}
\int_{0}^{y} p_{Y}(z) d z & =\text { probability that } 0 \leq Y \leq y \\
& =\text { probability that } 0 \leq X \leq T^{-1}(y) \\
& =\int_{0}^{T^{-1}(y)} p_{X}(w) d w
\end{aligned} \\
& \frac{d}{d y}\left(\int_{0}^{y} p_{Y}(z) d z\right)=p_{Y}(y)=p_{X}\left(T^{-1}(y)\right) \frac{d}{d y}\left(T^{-1}(y)\right)
\end{aligned}
$$

Our discrete histogram is an approximation of $p_{X}(x)$ and the transformation in Equation 2 approximates the one in Equation 4. While the discrete version won't result in exactly flat histograms, it will flatten them and in doing so enhance the contrast in the image.

The above describes the histogram equalization of the gray scale image. However, it can also be used on color images by applying the same method separately to the Red, Green and Blue components of the RGB color values of the image.

Fig.3(b) is the result of the histogram equalizing the Fig.3(a). Fig.3(c) is a histogram of Fig.3(a), Fig.3(d) is a histogram of Fig.3(c). When the Fig.2(b) histogram of level correction become histogram of Fig.3(d). The maximum value of the concentration of Fig.3 (c) is placed in 255 of the horizontal axis in Fig.3(d). In Fig.3(d), the histogram is stretched than the original image, a gap is generated between the histogram so intensity levels are rearranged.

\subsection{Conversion of coordinates}

Converted as a method of extracting a predetermined 


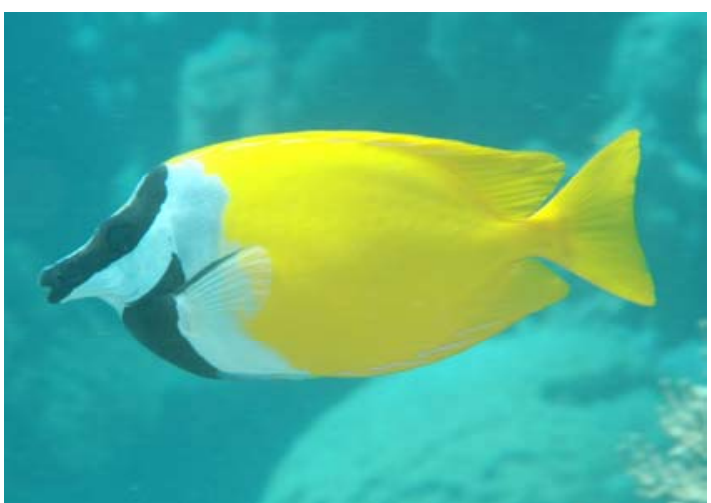

(a) Color cast image
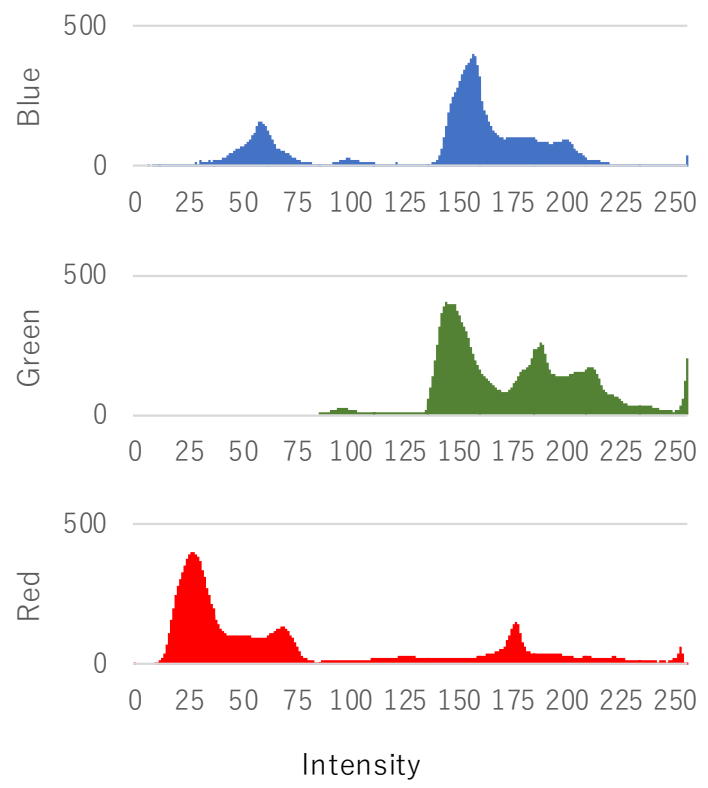

(c) Histogram of Color cast image.

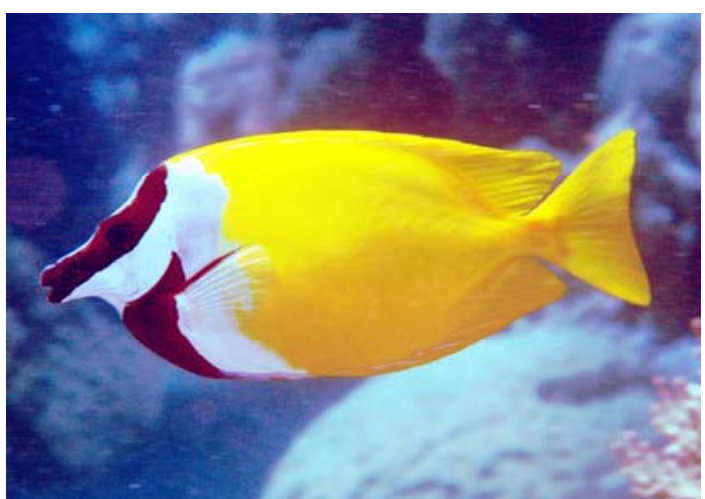

(b) Corrected image.
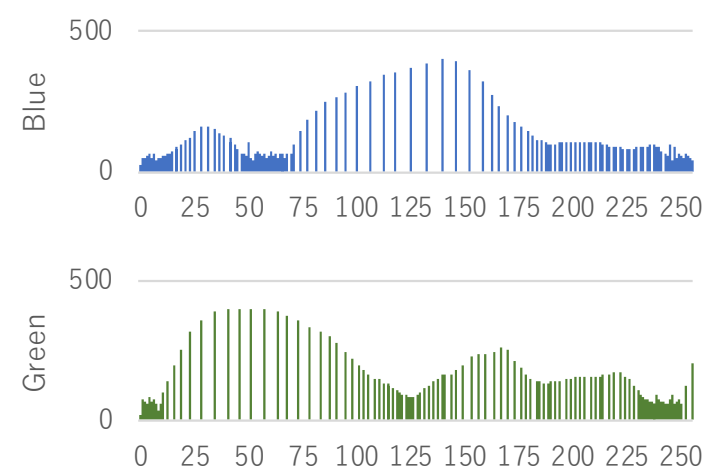

500

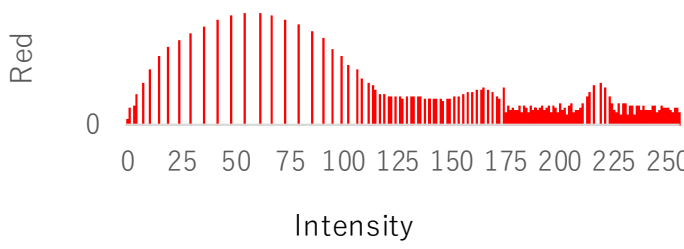

(d) Histogram of Corrected image

Fig. 3. Histogram Equalization

range from the image of the RGB coordinates is a color information in an HSV coordinates, specifying the local range within the range of the predetermined threshold. RGB coordinates is Red, Green, is a coordinates that has been composed of three color components of the Blue. Meanwhile, HSV coordinates, the hue (Hue), saturation (Saturation · Chroma), which is a coordinates composed of three components lightness (Value $\cdot$ Lightness $\cdot$ Brightness).

For RGB coordinates that consists of three color components of red, green and blue, it will generally have lower intensity values in a dark area, the higher the value the overall strength in a bright area. Across the bright and dark area in the RGB coordinates, to the detection of the same color must be set the value that contains the unwanted color gamut as a threshold value. As a result, it would also detect areas with unwanted color gamut. Meanwhile, HSV coordinates, the hue (Hue), saturation (Saturation · Chroma), which is a coordinates composed of three components lightness (Value · Lightness · Brightness). The color information in the HSV coordinates is managed by a single Hue, be different brightness becomes possible to point to a specific color gamut.

\section{Experimental Results and discussion}

In Section 3.1 shows the result of applying the histogram equalization in the local area. In Section 3.2 shows the performance of improving visibility of the stars of the proposed method. 


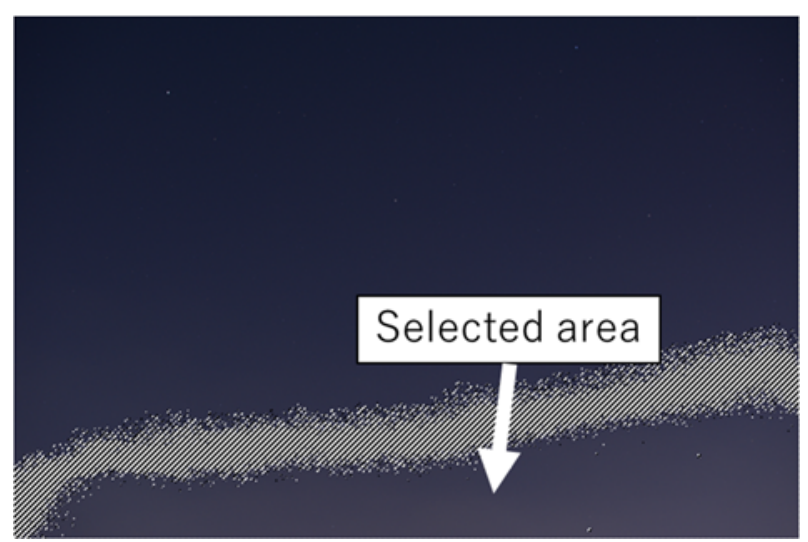

(a) Selected area

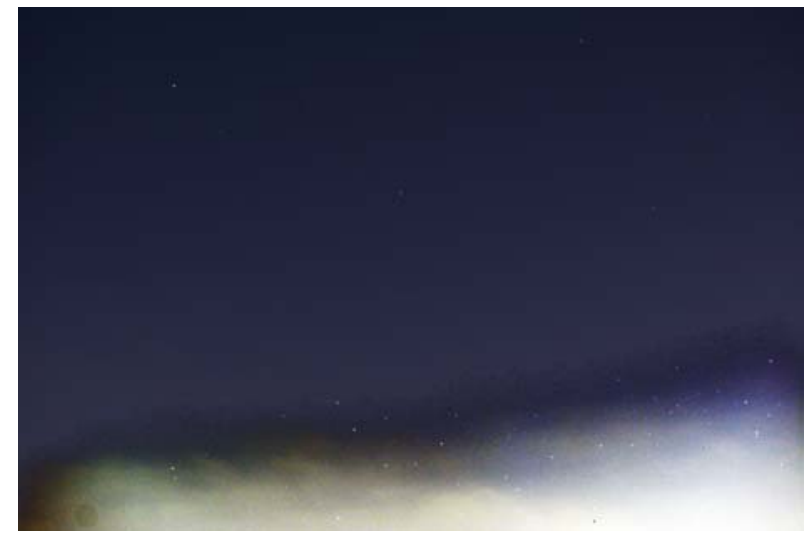

(b) Corrected image (local area)

Fig. 4. Color cast correction of the local area

\subsection{Color cast correction of the local area}

A color cast at the bottom of the image has been corrected by the proposed method for the image that as shown in Fig. 1(a). The area was selected by the proposed method is shown in Fig. 4(a). Shows the results of color correction by histogram equalization for the selected local area to Fig.4(b). The bottom left area of Fig.4(b) is different from the brightness of the background and stars. Consequently, visibility of the stars at the bottom left area of Fig.4(b) is higher than that of Fig.1(a) and Fig.1(b).

\subsection{Comparison of the visibility of the stars}

In the original images (Fig. 5(a), (d), (g), (j)) and the local area histogram equalization images (Fig. 5(b), (e), (h), (k)) and the entire histogram equalization images (Fig. 5(c), (f), (i), (1)), we compared the visibility of the stars. Fig.5 is an image set of the extracted $600 * 600$ pixels from the image of $6000 * 4000$ pixels. Table 1 is a result of manually

Table 1. The number of visually recognized stars

\begin{tabular}{|c|c|c|c|}
\hline & $\begin{array}{c}\text { Original } \\
\text { Image }\end{array}$ & $\begin{array}{c}\text { Histogram } \\
\text { Equalized } \\
\text { Image } \\
\text { (Entire Area) }\end{array}$ & $\begin{array}{c}\text { Histogram } \\
\text { Equalized } \\
\text { Image } \\
\text { (Local Area) }\end{array}$ \\
\hline Image 1 & 0 & 1 & 6 \\
\hline Image 2 & 2 & 10 & 14 \\
\hline Image 3 & 0 & 1 & 4 \\
\hline Image 4 & 0 & 3 & 5 \\
\hline
\end{tabular}

counting the number of visible Stars of each image of Fig. 5 . Table 1 shows that the visibility of the local area histogram equalization image is the best. When the histogram equalization of entire area, the entire image becomes brighter. Then, close the brightness of the night sky and stars as the background color. Therefore, improvement in the visibility of the histogram equalization of entire area is limited.

\section{Conclusions}

We have proposed a method to improve the visibility of the stars in the image with a brightness gradient. A local area of the image selected using threshold of the HSV coordinates by user. We have improved the visibility of the stars using the histogram equalization of local area. In the experiment, it was confirmed to improve the visibility by applying the proposed method to the image having the light gradation. Although the proposed method was able to correct the selected local region, false contour occurs in the boundary of the correction area and the non-correction area.

The proposed method, because the human intervention in the selection of the threshold and the local area is required, those of automation is the future. 


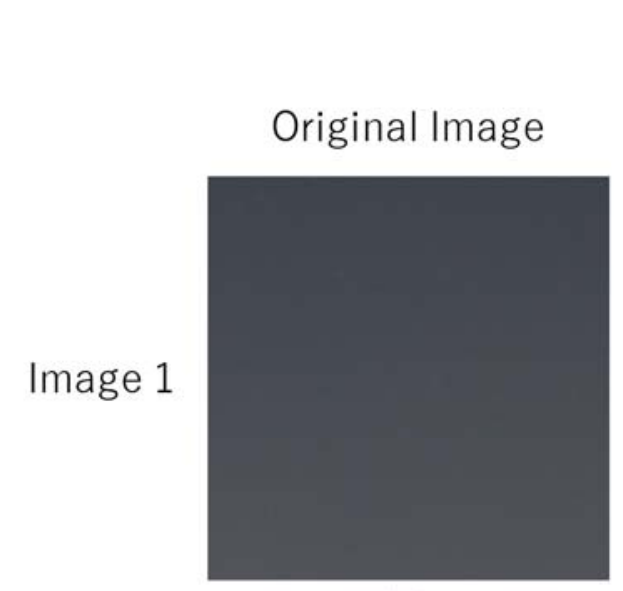

(a)

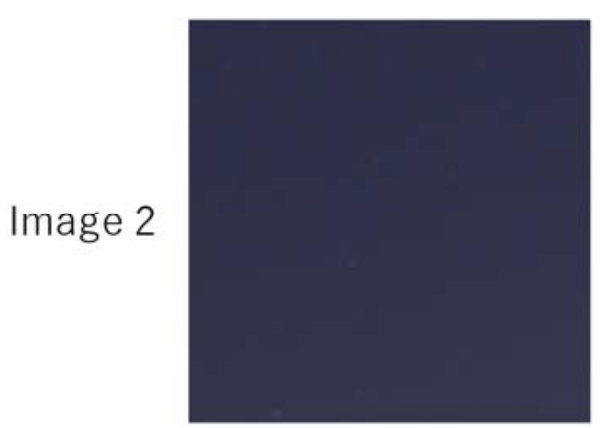

(d)

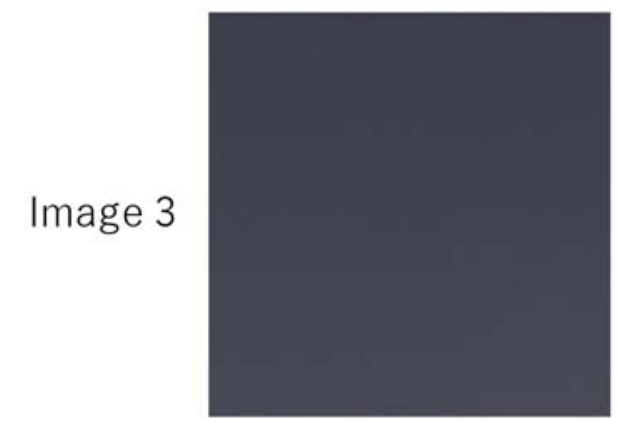

(g)

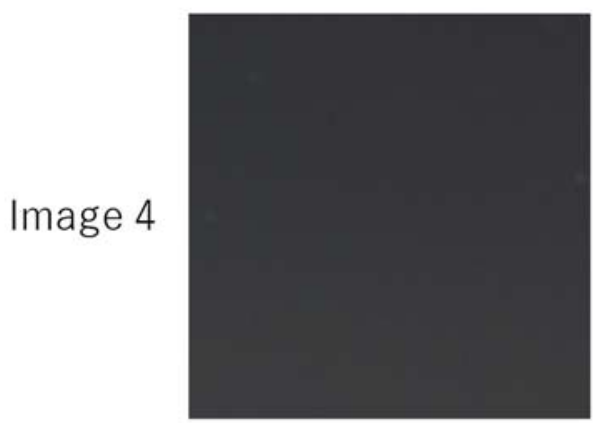

(j)
Histogram

Equalized Image

(Entire Area)

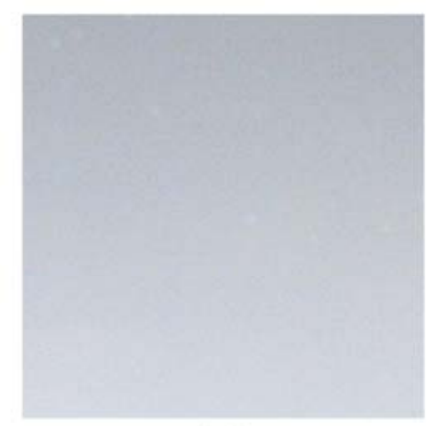

(b)

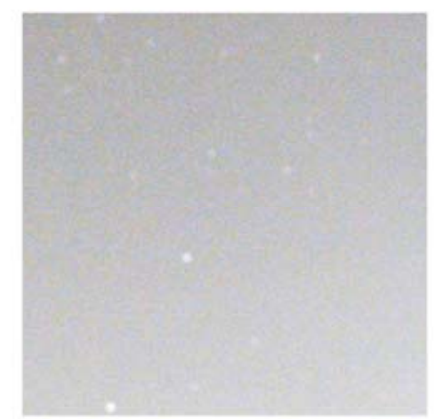

(e)

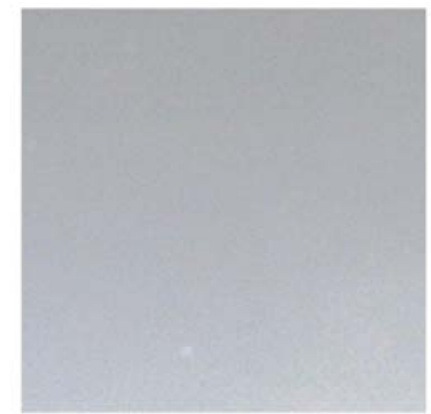

(h)

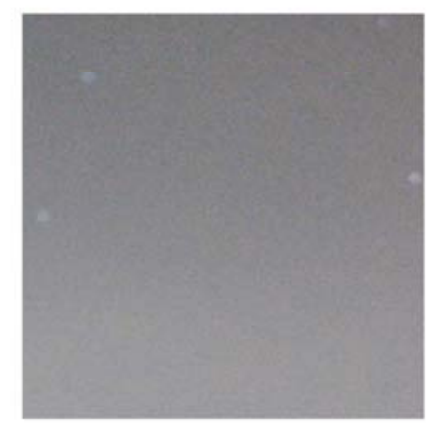

(k)
Histogram

Equalized Image

(Local Area)

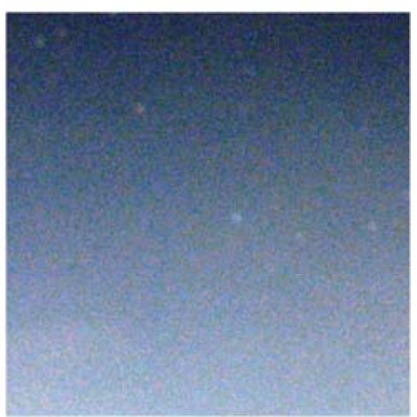

(c)

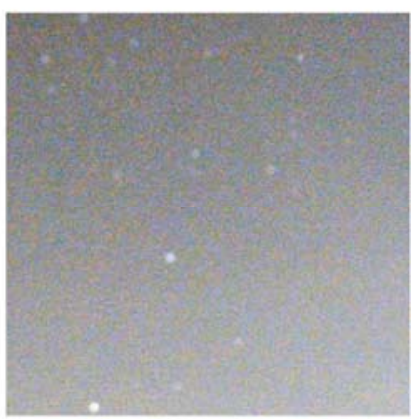

(f)

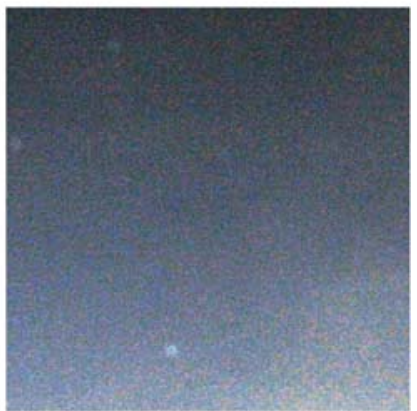

(i)

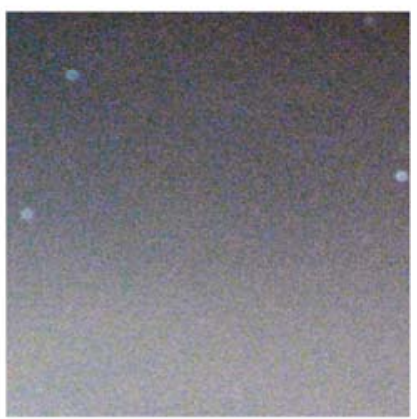

(I)

Fig. 5. Starry sky images 


\section{Acknowledgment}

This research was partially supported by a Grant-in-Aid for Scientific Research (Grant Nos. 26420770 and 23560913). The authors gratefully acknowledge the Japan Food Chemical Research Foundation (2016).

\section{References}

(1) K. Barnard, V. Cardei, and B. Funt, "A comparison of computational color constancy algorithms - part I: methodology and experiment with synthesized data," IEEE Trans. on Image Processing, vol. 11, pp. 985-996, Sept. 2002.

(2) K. Barnard, V. Cardei, and B. Funt, "A comparison of computational color constancy algorithms - part II: experiments with image data, "IEEE Trans. on Image Processing, vol. 11, pp. 985-996, Sept. 2002.

(3) K. Barnard, Practical color constancy, $\mathrm{PhD}$ thesis, Simon Fraser University, School of Computing, 1999.

(4) G.D. Finlayson, S.D. Hordley, and P.M. Hubel, "Color by correlation: a simple, unifying framework for color constancy," IEEE Trans. On Pattern Analysis and Machine Intelligence, vol. 23, pp. 1209-1221, Nov. 2001.

(5) R.C. Gonzales, and R.E. Woods, Digital Image Processing, Addison Wesley, Reading, MA, 1992.

(6) Y. T. Kim, "Contrast Enhancement Using Brightness Preserving Bi-Histogram Equation”, IEEE Transactions on Consumer Electronics, vol. 43, no. 1, February 1997, pp. 1-8.

(7) R. C. Gonzalez and R. E. Woods, "Digital Image Processing", 2nd edition, MA. Addison-Wesley, 1992, pp. 85-103.

(8) J. Zimmerman, S. Pizer, E. Staab, E. Perry, W. McCartney and B. Brenton, "Evaluation of the effectiveness of adaptive histogram equalization for contrast enhancement", IEEE Transaction on Medical Imaging, 1988, pp. 304-312.

(9) T. K. Kim, J. K. Paik and B. S. Kang, "Contrast enhancement system using spatially adaptive histogram equalization with temporal filtering”, IEEE Transaction on Consumer Electronics, vol. 44, no. 1, 1998, pp. 8286.

(10)R. C. Gonzalez and P. Wints, "Digital Image Processing", 2nd edition, Ed., Massachusetts: AddisonWesley Publishing Co., Reading, 1987. 